\title{
Lung cancer susceptibility from GSTM1 deletion and air pollution with smoking status: a meta-prediction of worldwide populations
}

This article has been corrected. Correction in Oncotarget. 2018; 9:36251-36251.

Pojui Yu ${ }^{1,2}$, Joyce D. Kusuma ${ }^{3}$, Maria Aurora R. Suarez ${ }^{4}$ and Shyang-Yun Pamela Koong Shiao ${ }^{5}$

\footnotetext{
${ }^{1}$ Department of Nursing, Fu Jen Catholic University Hospital, New Taipei City, Taiwan (R.O.C.)

${ }^{2}$ School of Nursing, College of Medicine, National Taiwan University, Taipei, Taiwan (R.O.C.)

${ }^{3}$ Heritage Victor Valley Medical Group, Big Bear Lake, CA, USA

${ }^{4}$ Department of Critical Care and Telemetry, Citrus Valley Health Partners, West Covina, CA, USA

${ }^{5}$ College of Nursing and Medical College of Georgia, Augusta University, Augusta, GA, USA

Correspondence to: Shyang-Yun Pamela Koong Shiao, email: pshiao@msn.com
}

Keywords: Glutathione S transferase mu 1; lung cancer; meta-prediction; air pollution; smoking

Received: May 15, $2018 \quad$ Accepted: June 13, $2018 \quad$ Published: July 24, 2018

Copyright: Yu et al. This is an open-access article distributed under the terms of the Creative Commons Attribution License 3.0 (CC BY 3.0), which permits unrestricted use, distribution, and reproduction in any medium, provided the original author and source are credited.

\section{ABSTRACT}

Glutathione S transferase mu 1 (GSTM1) gene has been associated with lung cancer (LC) risk, for GSTM1 enzyme playing a vital role in detoxification pathway and protective against toxic insults. The major objective of this study was to investigate GSTM1 deletion pattern and its association with LC in the world's population by using meta-prediction techniques. The secondary objective was to examine the effects of air pollution, smoking status, and other factors for gene-environment interactions with GSTM1 deletion and LC risk. We completed a comprehensive search to yield a total of 170 studies (40,296 cases and 48,346 controls) published from 1999 to 2017 for metaanalyses. The results revealed that GSTM1 deletion type was associated with increased risk of LC, while GSTM1 present type provided protective effect for all populations combined worldwide. Subgroup analysis on the rank order of risks from highest to lowest, among racial-ethnic groups, were Chinese, South East Asian, other North Asian, European, and finally American. Additional predictive analyses presented that air pollution played a significant role with increased risks of GSTM1 deletion and LC susceptibility, and the risks increased for smokers with higher levels of air pollution. Based on the findings of meta-predictive analysis, increased air pollution levels and smoking status presented additive effects to the LC risk susceptibilities and GSTM1 gene polymorphisms, for gene-environment interactions. Future studies are needed to examine gene-environment interactions for GSTM1 interacting with environmental factors and dietary interventions to mitigate the toxic effects, for LC prevention.

\section{INTRODUCTION}

Lung cancer (LC) accounts for the second most commonly diagnosed cancer among adults and $25 \%$ of all cancer deaths, with delayed diagnosis at a late stage being associated with poor prognosis [1-4]. Glutathione $S$ transferase mu 1 (GSTM1) gene has been associated with LC risk, with GSTM1 enzyme playing a vital role in detoxification pathway and protective effect against toxic insults [2, 5-7]. GSTM1 is one of phase II detoxification enzymes that detoxify electrophilic compounds, including carcinogens, therapeutic drugs, environmental toxins, and byproducts of oxidative stress by conjugation with glutathione (GSH). GSTM1 gene was known to be highly polymorphic and the polymorphism affects the expression of enzyme levels [5-11]. Two identified variants in GSTM1 are a deletion and a substitution. A deletion of GSTM1 or null mutation deactivates the enzymes, which results in the loss of function within the detoxification pathway [2-4]. GSTM1 null genotype has been associated 
with increased risk of many cancers [8], and increased environmental toxins and carcinogens further increase the susceptibility of LC [2, 4, 5, 7, 12].

Environmental toxicants such as air pollution and smoking can expose lung, an organ, to oxidative stress and dis-regulate reactive oxygen species [2, 4, 5, 13-15]. Studies suggested that exposure to oxidative stress cause damage to cellular DNA that leads to mutations, genomic instability, and ultimately malignancy $[2-4,13,14,16]$. Several studies indicated that consumption of cruciferous vegetables can reduce the risk of LC. These plants contain isothiocyanates (ITC) and indole-3-carbinol, which are known to induce phase II enzyme in the detox pathway $[14,17,18]$. ITC and indoles may inhibit the bioactivation of carcinogen from air pollution and smoke, enhance excretion of carcinogenic metabolites before it causes damage to DNA, and induce cell cycle arrest and apoptosis $[18,19]$. These processes affirm the crucial role of micronutrients in the detoxification pathway for LC prevention.

To date, results from epidemiological studies on the association of GSTM1 mutation and LC have been inconsistent and mixed with heterogeneous findings. Meta-predictive analysis can be used to address heterogeneous findings, and to cross validate the findings using various analytical methods [20]. Additional studies indicated the effects of air pollution on the association with GSTM1 deletion. Despite these findings, previous metaanalyses did not examine the effects of gene-environment interaction, specifically air pollution and smoking status, on the association with GSTMI and LC risk. To fill this gap and to provide further evidence, we conducted a meta-analysis by adding meta-predictive techniques to examine the impact of exposure to air pollution on the risk of GSTM1 deletion and LC susceptibility in various populations of the world, with subgroup analyses of LC types, smoking status, and gender status. In this meta-prediction study, we integrated the use of big-data machine-learning analytics in addition to the conventional pooled analysis, including the global maps and heat maps to visualize grouping patterns.

\section{RESULTS}

\section{Characteristics of study subjects}

We have summarized how we selected studies in Figure 1. We initially identified 450 potential relevant studies published from 1999 to 2017 . Through systematic screening process, we located a total of 163 papers $(40,296$ cases and 48,346 controls) that included data for GSTM1 deletion. These studies were conducted in 5 continents of the world and 7 studies also included data for more than one racial-ethnic groups, yielding a total of 170 studies (see Supplementary Table 1, see Figure 2 for \% GSTM1 deletion in control and LC groups).

\section{Pooled analysis - by ethnic groups}

For all included studies, Table 1 (summary schema) and Table 2 (detailed pooled analyses) presented increased risk of LC with GSTM1 deletion ( $\mathrm{RR}=1.10, p<0.0001)$, while GSTM1 present genotype were protective against $\mathrm{LC}(\mathrm{RR}=0.91, p<0.0001)$. Subgroup analysis per ethnic groups showed the rank order of highest risk of LC with GSTM1 deletion, being among Chinese $(\mathrm{RR}=1.20$, $p<0.0001)$, South East Asian $(\mathrm{RR}=1.12, p=0.0165)$, other North Asian $(\mathrm{RR}=1.08, p<0.0001)$, European $(\mathrm{RR}=1.06, p=0.0005)$, and finally American $(\mathrm{RR}=1.04$, $p=0.02)$. There were no significances on the risks for the three additional ethnic subgroups of Oceanian (2 studies), African American (8 studies), and mixed ethnic groups (8 studies).

\section{Subgroup analyses by LC type per total population and ethnic groups}

Per LC subtypes, Table 1 presented that the risks of LC were similar for different LC types with GSTM1 deletion for all populations combined (non-small cell LC [NSCLC]: RR $=1.11, p<0.0001$; Mixed LC types: $\mathrm{RR}=1.10, p<0.0001)$. For ethnic subgroup analyses per LC types, the risk of LC for Chinese was slightly lower in NSCLC subtype than the mixed LC subtype (NSCLC: $\mathrm{RR}=1.17, p<0.0001$; mixed LC: $\mathrm{RR}=1.21, p<0.0001)$ (see Supplementary Table 2). Additionally, significant risks were noted for mixed LC type in north Asian $(\mathrm{RR}=$ $1.09, p<0.0001)$ and European $(\mathrm{RR}=1.06, p=0.0007)$.

\section{Subgroup analyses by smoking and gender status per total population and ethnic groups}

Per smoking status (Table 1), the risk of LC was mixed and presented inconsistent findings across ethnic subgroups. The risk was slightly higher for non-smokers $(\mathrm{RR}=1.15, p<0.0001)$ than smokers $(\mathrm{RR}=1.09$; $p<0.0001)$. However, the reversed findings were noted among Chinese (smokers: $\mathrm{RR}=1.27, p<0.0001$; nonsmokers: $\mathrm{RR}=1.22, p<0.0001$ ) (see Supplementary Table 3). There were no significances for subgroup analyses of smoking status for other racial-ethnic subgroups.

The risk of LC was also mixed and presented inconsistent findings across gender subgroups (Table 1). The risk was slightly higher for female $(\mathrm{RR}=1.13$, $p=0.0003)$ than male $(\mathrm{RR}=1.08, p=0.0045)$. Similar risks of LC are noted in other North Asian (female: $\mathrm{RR}=1.21, p=0.0403$; male: $\mathrm{RR}=1.08, p=0.0039$ ). However, the reversed findings were noted among South East Asian male when compared to female (male: $\mathrm{RR}=$ $1.57, p=0.0004$; female: $\mathrm{RR}=1.36, p=0.0006$ ) (see Supplementary Table 4). No significant findings were present for subgroup analyses on gender status of other racial ethnic groups. 


\section{Subgroup analyses by countries}

To identify sources of heterogeneity, we further performed subgroup analyses per countries using geographic information system (GIS) to visualize regional distributions and to validate the heterogeneity of the findings. Countries were divided based on geographical area. These geographical analyses showed the rank order of highest risk of LC with GSTM1 deletion, being Chinese, South East Asian, other North Asian, European countries and American countries (Table 2, Supplementary Figure 1A-1D). The global maps demonstrated the variations in the GSTMI deletion and their LC risk susceptibilities across regions. In the first two GIS maps, we used the continuous color spectrum from yellow to red, representing the increasing levels of polymorphisms, and in the third map, red-green colors - red indicating LC risk, and green indicating protective effects. Similar to the pooled meta-analysis, GIS maps showed that GSTM1 deletion played a risk role in LC in most countries except Australia, Pakistan, Poland, Sweden, Italy, United Kingdom (UK) and Portugal (Supplementary Figure 2).

\section{Meta-prediction}

Given the heterogeneous findings on the effects of GSTM1 deletion and the risk susceptibility of LC, we performed meta-predictive analysis using both big-data machine-learning predictive analytics and conventional analyses (Table 3). We used both partition tree and Tukey's tests to examine the potential interaction between air pollution and deletions, and their impact on LC risks. Based on the guidelines from the World Health Organization on air pollution, we used the levels of death from air pollution (APD) as the measure of air quality (Level 2: 51-100, Level 3: 101-250, and Level 4: > 251 deaths/million) [33-38]. The partition tree and Tukey's test results converged and showed significant differences between APD Levels 3 and $4(p<0.0001)$, and between Levels 2 and $4(p=0.0056)$ for percent GSTM1 deletion by APD for LC cases. The same trend of statistical significance was noted on GSTM1 present type for LC cases. Furthermore, on the risk for GSTM1 deletion, significant differences were identified between Levels 3 and $4(p=0.0479)$, with the smallest AICc of -24.28 .

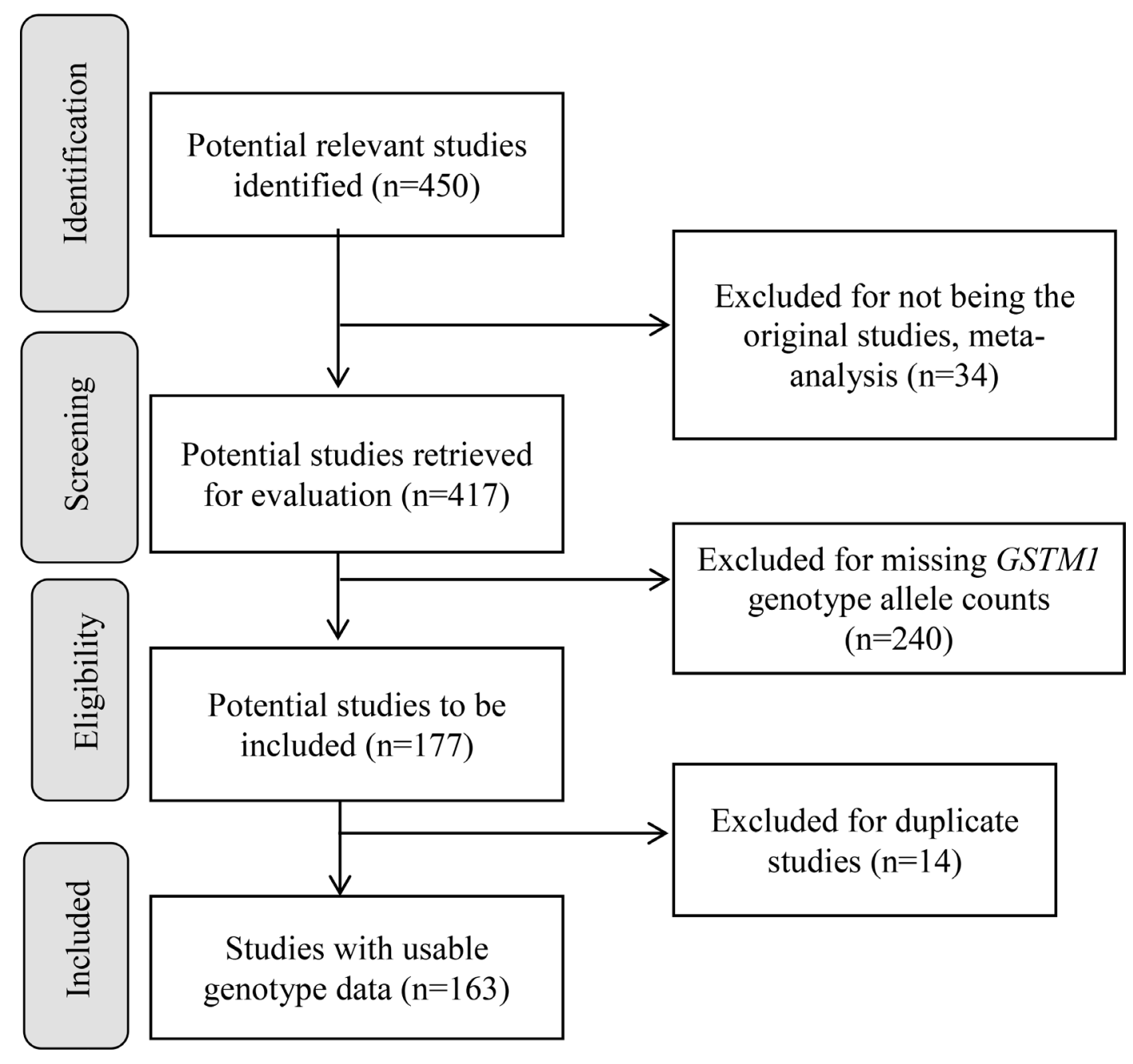

Figure 1: Progression on the selection of studies for the meta-analysis. 
Table 1: Schema of significant findings on GSTM1 deletion and risk of lung cancer per ethnic subgroups $(n=170)$

\begin{tabular}{|c|c|c|c|c|c|c|c|c|c|}
\hline \multirow{2}{*}{$\begin{array}{l}\text { GSTM1 } \\
\text { (n Case/ } \\
\text { n Control) }\end{array}$} & \multirow{2}{*}{$\begin{array}{c}\text { Deletion } \\
\text { (n Case/n Control) }\end{array}$} & \multicolumn{2}{|c|}{ LC Type } & \multicolumn{3}{|c|}{ Smoker Status ${ }^{A}$} & \multicolumn{3}{|c|}{ Gender $^{\mathrm{B}}$} \\
\hline & & NSCLC & Mixed & Yes & No & NA & Male & Female & NA \\
\hline $\begin{array}{l}\text { All } \\
(40,236 / 48,200)\end{array}$ & $\begin{array}{c}170 \text { Studies } \\
(21,248 / 23,513) \\
\text { RR } 1.10\end{array}$ & $\begin{array}{c}38 \text { Studies } \\
(4,231 / 4,132) \\
\text { RR } 1.11\end{array}$ & $\begin{array}{c}132 \text { Studies } \\
(17,050 / 19,440) \\
\text { RR } 1.10\end{array}$ & $\begin{array}{c}63 \text { Studies } \\
(7,776 / 6,756) \\
\text { RR } 1.09\end{array}$ & $\begin{array}{c}49 \text { Studies } \\
(1,513 / 3,494) \\
\text { RR } 1.15\end{array}$ & $\begin{array}{c}\text { 104 Studies } \\
(11,508 / 12,430) \\
\text { RR } 1.11\end{array}$ & $\begin{array}{c}25 \text { Studies } \\
(5,072 / 3,883) \\
\text { RR } 1.08\end{array}$ & $\begin{array}{c}26 \text { Studies } \\
(2,221 / 3,165) \\
\text { RR } 1.13\end{array}$ & $\begin{array}{c}139 \text { Studies } \\
(13,774 / 16,269) \\
\text { RR } 1.11\end{array}$ \\
\hline \multicolumn{10}{|l|}{ European } \\
\hline$(13,386 / 17,012)$ & $\begin{array}{l}48 \text { Studies } \\
(6,915 / 8,372) \\
\text { RR } 1.06\end{array}$ & $\begin{array}{c}7 \text { Studies } \\
(497 / 630) \\
\text { NS }\end{array}$ & $\begin{array}{l}41 \text { Studies } \\
(6,418 / 7,742) \\
\text { RR } 1.06\end{array}$ & $\begin{array}{c}18 \text { Studies } \\
(3,766 / 3,400) \\
\text { NS }\end{array}$ & $\begin{array}{c}12 \text { Studies } \\
\text { (323/1,277) } \\
\text { NS }\end{array}$ & $\begin{array}{c}28 \text { Studies } \\
(2,444 / 3,108) \\
\text { RR } 1.07\end{array}$ & $\begin{array}{c}11 \text { Studies } \\
(2,160 / 2,303) \\
\text { NS }\end{array}$ & $\begin{array}{c}10 \text { Studies } \\
(555 / 1,058) \\
\text { NS }\end{array}$ & $\begin{array}{c}37 \text { Studies } \\
(4,460 / 5,087) \\
\text { RR } 1.08\end{array}$ \\
\hline \multicolumn{10}{|l|}{ Oceanian } \\
\hline$(1,541 / 1,101)$ & $\begin{array}{l}2 \text { Studies } \\
(865 / 632) \\
\text { NS }\end{array}$ & $\begin{array}{l}1 \text { Study } \\
(591 / 367)\end{array}$ & $\begin{array}{l}\text { 1 Study } \\
(274 / 265)\end{array}$ & $\begin{array}{l}\text { 1 Study } \\
(591 / 367)\end{array}$ & -- & $\begin{array}{l}1 \text { Study } \\
(274 / 265)\end{array}$ & $\begin{array}{c}1 \text { Study } \\
(420 / 247)\end{array}$ & $\begin{array}{l}\text { 1 Study } \\
(171 / 120)\end{array}$ & $\begin{array}{l}\text { 1 Study } \\
(274 / 265)\end{array}$ \\
\hline \multicolumn{10}{|l|}{ American } \\
\hline$(5,350 / 7,828)$ & $\begin{array}{l}18 \text { Studies } \\
(2,876 / 4,026) \\
\text { RR } 1.04\end{array}$ & $\begin{array}{l}\text { 4 Studies } \\
\text { (798/820) } \\
\text { NS }\end{array}$ & $\begin{array}{c}14 \text { Studies } \\
(2,078 / 3,206) \\
\text { NS }\end{array}$ & $\begin{array}{c}8 \text { Studies } \\
(1,094 / 1,329) \\
\text { NS }\end{array}$ & $\begin{array}{l}5 \text { Studies } \\
(61 / 509) \\
\text { NS }\end{array}$ & $\begin{array}{c}10 \text { Studies } \\
(1,695 / 2,063) \\
\text { NS }\end{array}$ & $\begin{array}{l}1 \text { Study } \\
(35 / 95)\end{array}$ & $\begin{array}{l}2 \text { Studies } \\
(250 / 324) \\
\text { NS }\end{array}$ & $\begin{array}{c}16 \text { Studies } \\
(2,591 / 3,607) \\
\text { NS }\end{array}$ \\
\hline \multicolumn{10}{|l|}{ Mixed } \\
\hline$(1,537 / 2,510)$ & $\begin{array}{l}8 \text { Studies } \\
(768 / 1,216) \\
\text { NS }\end{array}$ & -- & $\begin{array}{l}8 \text { Studies } \\
(768 / 1,216) \\
\text { NS }\end{array}$ & $\begin{array}{l}\text { 1 Study } \\
(136 / 171)\end{array}$ & $\begin{array}{l}1 \text { Study } \\
(7 / 74)\end{array}$ & $\begin{array}{l}7 \text { Studies } \\
(625 / 970) \\
\text { NS }\end{array}$ & $\begin{array}{l}1 \text { Study } \\
(53 / 35)\end{array}$ & $\begin{array}{l}1 \text { Study } \\
(50 / 63)\end{array}$ & $\begin{array}{l}7 \text { Studies } \\
(665 / 1,118) \\
\text { NS }\end{array}$ \\
\hline \multicolumn{10}{|l|}{ African American } \\
\hline$(790 / 1,024)$ & $\begin{array}{l}8 \text { Studies } \\
(233 / 258) \\
\text { NS }\end{array}$ & $\begin{array}{l}1 \text { Study } \\
(36 / 28)\end{array}$ & $\begin{array}{l}\text { 7 Studies } \\
(197 / 230) \\
\text { NS }\end{array}$ & $\begin{array}{l}1 \text { Study } \\
(33 / 22)\end{array}$ & $\begin{array}{c}1 \text { Study } \\
(3 / 2)\end{array}$ & $\begin{array}{l}7 \text { Studies } \\
(196 / 234) \\
\text { NS }\end{array}$ & -- & $\begin{array}{l}1 \text { study } \\
(36 / 28)\end{array}$ & $\begin{array}{c}7 \text { Studies } \\
(197 / 230) \\
\text { NS }\end{array}$ \\
\hline \multicolumn{10}{|l|}{ Mexican } \\
\hline$(60 / 146)$ & $\begin{array}{l}\text { 1 Study } \\
(33 / 59)\end{array}$ & -- & $\begin{array}{l}1 \text { study } \\
(33 / 59)\end{array}$ & -- & -- & $\begin{array}{l}1 \text { study } \\
(33 / 59)\end{array}$ & -- & -- & $\begin{array}{l}1 \text { study } \\
(33 / 59)\end{array}$ \\
\hline \multicolumn{10}{|l|}{ North Asian } \\
\hline$(7,432 / 5,888)$ & $\begin{array}{c}18 \text { Studies } \\
(4,159 / 3,006) \\
\text { RR } 1.08\end{array}$ & $\begin{array}{c}5 \text { Studies } \\
(578 / 774) \\
\text { NS }\end{array}$ & $\begin{array}{c}13 \text { Studies } \\
(3,581 / 2,232) \\
\text { RR } 1.09\end{array}$ & $\begin{array}{l}7 \text { Studies } \\
(685 / 509) \\
\text { RR } 1.18\end{array}$ & $\begin{array}{c}5 \text { Studies } \\
(211 / 335) \\
\text { NS }\end{array}$ & $\begin{array}{c}10 \text { Studies } \\
(3,232 / 2,088) \\
\text { RR } 1.06\end{array}$ & $\begin{array}{c}\text { 6 Studies } \\
(2,439 / 1,034) \\
\text { RR } 1.08\end{array}$ & $\begin{array}{l}\text { 4 Studies } \\
(621 / 710) \\
\text { RR } 1.21\end{array}$ & $\begin{array}{c}11 \text { Studies } \\
(1,099 / 1,262) \\
\text { RR } 1.08\end{array}$ \\
\hline \multicolumn{10}{|l|}{ Chinese } \\
\hline$(6,932 / 8,974)$ & $\begin{array}{c}49 \text { Studies } \\
(3,992 / 4,452) \\
\text { RR } 1.20\end{array}$ & $\begin{array}{c}16 \text { Studies } \\
(1,483 / 1,338) \\
\text { RR } 1.17\end{array}$ & $\begin{array}{c}33 \text { Studies } \\
(2,509 / 3,114) \\
\text { RR } 1.21\end{array}$ & $\begin{array}{c}19 \text { Studies } \\
(1,154 / 749) \\
\text { RR } 1.27\end{array}$ & $\begin{array}{l}18 \text { Studies } \\
\text { (712/923) } \\
\text { RR } 1.22\end{array}$ & $\begin{array}{c}30 \text { Studies } \\
(2,162 / 2,798) \\
\text { RR } 1.19\end{array}$ & $\begin{array}{c}3 \text { Studies } \\
(268 / 340) \\
\text { NS }\end{array}$ & $\begin{array}{l}5 \text { Studies } \\
(418 / 689) \\
\text { NS }\end{array}$ & $\begin{array}{c}44 \text { Studies } \\
(3,306 / 3,423) \\
\text { RR } 1.20\end{array}$ \\
\hline \multicolumn{10}{|l|}{ South East Asian } \\
\hline$(3,268 / 3,863)$ & $\begin{array}{c}18 \text { Studies } \\
(1,407 / 1,492) \\
\text { RR } 1.12\end{array}$ & $\begin{array}{c}\text { 4 Studies } \\
(248 / 175) \\
\text { NS }\end{array}$ & $\begin{array}{c}14 \text { Studies } \\
(1,159 / 1,317) \\
\text { NS }\end{array}$ & $\begin{array}{c}8 \text { Studies } \\
(317 / 209) \\
\text { NS }\end{array}$ & $\begin{array}{c}7 \text { Studies } \\
(196 / 374) \\
\text { NS }\end{array}$ & $\begin{array}{c}10 \text { Studies } \\
\text { (847/845) } \\
\text { RR } 1.20\end{array}$ & $\begin{array}{l}2 \text { Study } \\
(117 / 76) \\
\text { RR } 1.57\end{array}$ & $\begin{array}{c}2 \text { Studies } \\
(120 / 173) \\
\text { RR } 1.36\end{array}$ & $\begin{array}{c}15 \text { Studies } \\
(1,149 / 1,218) \\
\text { RR } 1.08\end{array}$ \\
\hline
\end{tabular}

Note: GSTMI = Glutathione S transferase $m u$ 1; RR = relative risk; $\mathrm{LC}=$ lung cancer; NSCLC = non-small cell lung cancer; NA = not available; -- no data;

${ }^{\mathrm{A}} 46$ studies had data for both smoker and non-smoker groups; ${ }^{\mathrm{B}} 20$ studies had both male and female groups.

There were no significant differences based on gender status. To further illustrate the significance, we plotted those results on nonlinear curves. We noticed increased percentages of GSTM1 deletion for all groups of LC (Supplementary Figure 3A), NSCLC (Supplementary Figure 3B) and mixed LC type (Supplementary Figure 3C); and non-smoker groups (Supplementary Figure 3D) with the increased air pollution (Level 2: $\leq 100$, Level 3: 101-250, and Level 4: $\geq 251$ deaths/million). In contrast, the increase in deletion rates per air pollution levels were not as noticeable for the control groups. The results on the heat map were revealing for data density with the red blocks being the areas of high data concentration and the nonlinear fit line following the dense data (the red cells) for the percentages of GSTM1 deletion (Supplementary Figure $4 \mathrm{~A}-4 \mathrm{H}$ ) and LC risk (Supplementary Figure $5 \mathrm{~A}-5 \mathrm{D})$.
Higher percentages of GSTM1 deletion was also noted with the smoking status for smokers (Figure 3, left graph). We noticed obvious increased risks of LC for smokers with the increased air pollution from low levels (Level 2 and Level 3) to high level (Level 4) (Figure 3 right graph). Similar trends, however, no obvious increases of LC risks were noted for non-smokers or other LC types with GSTM1 deletion (Supplementary Figure $3 \mathrm{~A}-3 \mathrm{D})$. The most noteworthy finding is that with the increased air pollution levels, in smokers, the LC risk (Figure 3, right graph) was significantly higher in Level $4(\mathrm{RR}=1.25)$ than other two levels $(\mathrm{RR}=1.01)(p<0.05$ for both Tukey's tests between Level 4 versus Levels 3 and 2), based on GSTM1 deletion. These significantly increased LC risks for smokers at higher air pollution, contrary to not noticeable increases for other subgroups (Supplementary Figure 3A-3D), presented additive 
effects of gene-environment interactions based on GSTM1 deletion interacting with air pollution and smoking status. The results on the heat map were revealing for data density with the red blocks being the areas of high data concentration and the nonlinear fit line following the dense data (the red cells) for the percentages of GSTM1 deletion (Figure 4A, 4B) and LC risk (Figure 4C).

\section{DISCUSSION}

To date, previous studies presented the combined effects of GST family on the LC risks [21-26]. By using the meta-predictive analyses, we provided the most inclusive analyses of LC risk susceptibility based on GSTM1 deletion interacting with air pollution and smoking status. We completed a comprehensive search to yield a total of 170 studies (40,296 cases and 48,346 controls) published from 1999 to 2017 . The analyses by countries indicated increased GSTM1 deletion rates and LC risks in Asian countries. Subgroup analysis on the rank order of risks from highest to lowest, among racial-ethnic groups, were Chinese, South East Asian, other North Asian, European, and finally American. These studies were conducted around the globe and its continents (e.g., Australia, Europe, North and South America, and Asia).
The most investigated racial-ethnic populations for GSTM1 in association with LC was Asian (85 studies), Caucasian (68 studies), African (8 studies), Mexican (1 study), and mixed racial-ethnic groups ( 8 studies). Our results confirmed previous meta-analyses that GSTM1 deletion was associated with increased risk of LC [27-32], while GSTM1 present genotype provided protective effect.

Additional noteworthy findings from subgroup analyses showed that higher risk of LC was presented among non-smokers than smokers with GSTM1 deletion in worldwide populations combined. Conversely, smokers had higher risks of LC than non-smokers of LC with GSTMI deletion in Chinese subgroup. The findings about nonsmokers having overall higher risk of LC than smokers with GSTM1 deletion in our findings are consistent with a previous meta-analysis of Chinese populations [2]. However, we used risk ratios to standardize these risks (with the total counts as the denominator instead of one of the deletion or present type as the denominator) as contrary to the use of odds ratios (using one of the deletion or present type as the denominator) in previous meta-analyses. The standardized ratio is necessary when conducting gene-environment interactions across various factors for their standardized effects on the outcomes of polymorphism or LC risk [20, 33, 34]. The mechanism of higher LC risks from tobacco included

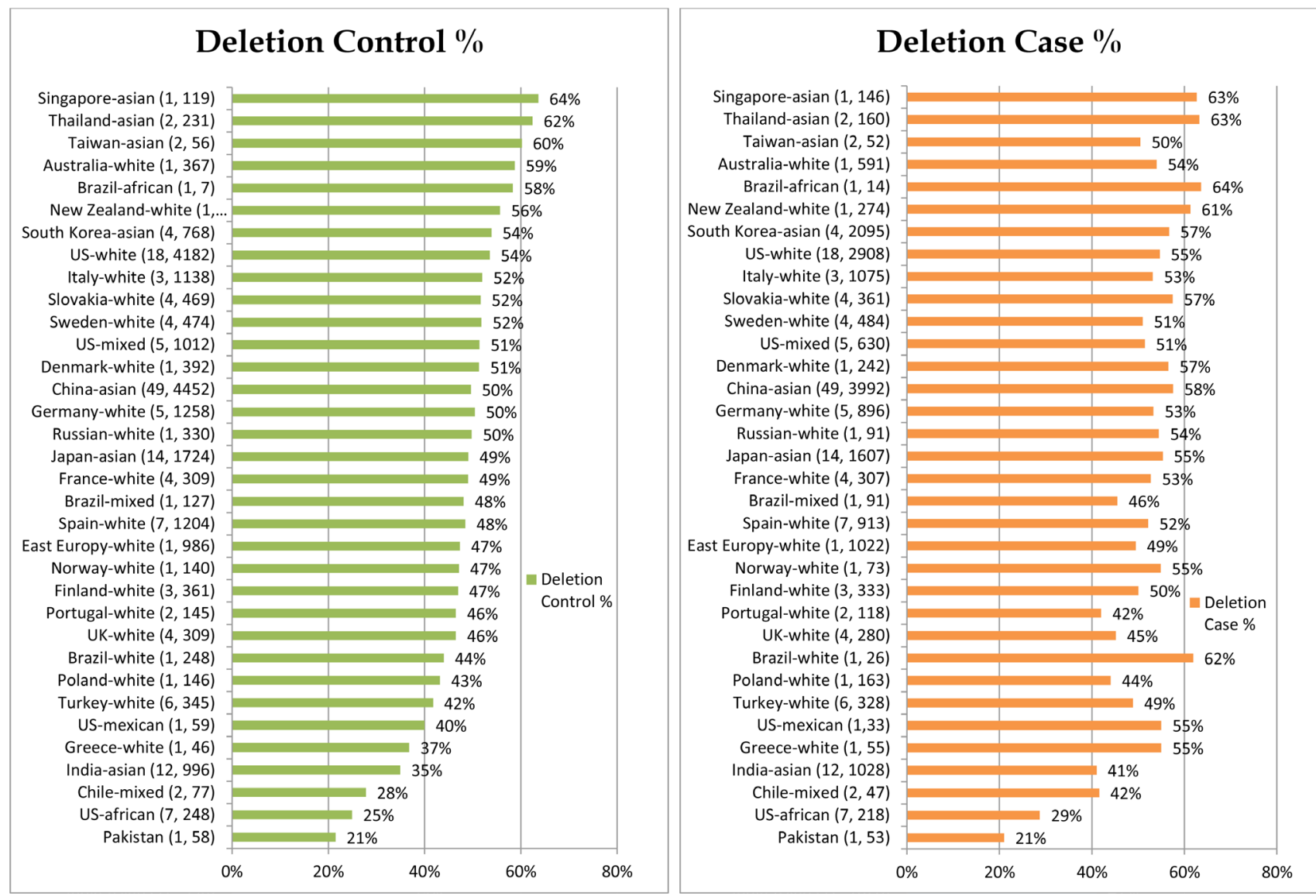

Figure 2: GSTM1 \% deletion per control and case groups. 
Table 2: Pooled analysis: GSTM1 deletion and risk of lung cancer

\begin{tabular}{|c|c|c|c|c|c|c|c|c|}
\hline \multirow{2}{*}{$\begin{array}{l}\text { Genotype } \\
\text { (number of studies) }\end{array}$} & \multirow{2}{*}{$\begin{array}{c}\text { LC Case } \\
N=40,296 \\
n(\%)\end{array}$} & \multirow{2}{*}{$\begin{array}{c}\text { Control } \\
\begin{array}{c}N=48,346 \\
n(\%)\end{array}\end{array}$} & \multicolumn{3}{|c|}{ Test of Heterogeneity } & \multirow{2}{*}{$\begin{array}{l}\text { Statistical } \\
\text { Model }\end{array}$} & \multicolumn{2}{|c|}{ Test of Association } \\
\hline & & & $\mathbf{Q}$ & $p$ & $I^{2}(\%)$ & & $\begin{array}{c}\text { Risk Ratio }(95 \% \\
\text { CI) }\end{array}$ & $p$ \\
\hline Deletion (170) & $21,248(52.7)$ & $23,513(48.6)$ & 356.76 & $<0.0001$ & $52.6 \%$ & Random & $1.10(1.08-1.13)$ & $<0.0001$ \\
\hline European (48) & $6,915(51.7)$ & $8,372(49.2)$ & 80.47 & 0.002 & $41.6 \%$ & Random & $1.06(1.03-1.10)$ & 0.0005 \\
\hline Oceanian (2) & $865(56.1)$ & $632(57.4)$ & 6.47 & 0.01 & $84.5 \%$ & Random & $1.00(0.84-1.20)$ & 0.97 \\
\hline American (18) & $2,876(53.8)$ & $4,026(51.4)$ & 11.92 & 0.80 & $0.0 \%$ & Fixed & $1.04(1.01-1.08)$ & 0.02 \\
\hline Mixed (8) & $768(50.0)$ & $1,216(48.4)$ & 11.68 & 0.11 & $40.1 \%$ & Fixed & $1.00(0.94-1.07)$ & 0.92 \\
\hline African American (8) & $233(29.5)$ & $258(25.2)$ & 3.62 & 0.82 & $0.0 \%$ & Fixed & $1.16(0.99-1.35)$ & 0.07 \\
\hline Mexican (1) & $33(55.0)$ & $59(40.4)$ & - & - & - & - & - & - \\
\hline North Asian (18) & $4,159(56.0)$ & $3,006(51.1)$ & 19.22 & 0.32 & $11.5 \%$ & Fixed & $1.08(1.05-1.12)$ & $<0.0001$ \\
\hline Chinese (49) & $3,992(57.6)$ & 4,452 (49.6) & 122.14 & $<0.0001$ & $60.7 \%$ & Random & $1.20(1.14-1.26)$ & $<0.0001$ \\
\hline South East Asian (18) & $1,407(43.1)$ & 1,492 (38.6) & 42.05 & 0.0007 & $59.6 \%$ & Random & $1.12(1.02-1.22)$ & 0.0165 \\
\hline Present (170) & $19,048(47.3)$ & $24,833(51.4)$ & 336.93 & $<0.0001$ & $49.8 \%$ & Random & $0.91(0.89-0.93)$ & $<0.0001$ \\
\hline European (48) & $6,471(48.3)$ & $8,640(50.8)$ & 71.96 & 0.01 & $34.7 \%$ & Random & $0.95(0.92-0.98)$ & 0.0006 \\
\hline Oceanian (2) & $676(43.9)$ & 469 (42.6) & 6.22 & 0.01 & $83.9 \%$ & Random & $0.99(0.78-1.26)$ & 0.95 \\
\hline American (18) & $2,474(46.2)$ & $3,802(48.6)$ & 10.82 & 0.87 & $0.0 \%$ & Fixed & $0.96(0.92-0.99)$ & 0.03 \\
\hline Mixed (8) & $769(50.0)$ & $1,294(51.6)$ & 8.75 & 0.27 & $20.0 \%$ & Fixed & $1.00(0.94-1.06)$ & 0.92 \\
\hline African American (8) & $557(70.5)$ & $766(74.8)$ & 3.71 & 0.81 & $0.0 \%$ & Fixed & $0.95(0.89-1.00)$ & 0.06 \\
\hline Mexican (1) & $27(45.0)$ & 87 (59.6) & - & - & - & - & - & - \\
\hline North Asian (18) & $3,273(44.0)$ & 2,882 (48.9) & 19.00 & 0.33 & $10.5 \%$ & Fixed & $0.91(0.88-0.95)$ & $<0.0001$ \\
\hline Chinese (49) & $2,940(42.4)$ & $4,522(50.4)$ & 117.76 & $<0.0001$ & $59.2 \%$ & Random & $0.82(0.77-0.86)$ & $<0.0001$ \\
\hline South East Asian (18) & $1,861(56.9)$ & 2,371 (61.4) & 41.09 & 0.0009 & $58.6 \%$ & Random & $0.93(0.87-0.99)$ & 0.0148 \\
\hline \multicolumn{9}{|c|}{ Subgroup of Deletion Type } \\
\hline \multicolumn{9}{|l|}{ LC type } \\
\hline NSCLC (38) & 4,167 (19.6) & 4,069 (17.3) & 85.03 & $<0.0001$ & $56.5 \%$ & Random & $1.11(1.06-1.17)$ & $<0.0001$ \\
\hline Mixed (132) & $17,050(80.4)$ & $19,440(82.7)$ & 272.89 & $<0.0001$ & $52.0 \%$ & Random & $1.10(1.07-1.13)$ & $<0.0001$ \\
\hline \multicolumn{9}{|l|}{ Smoker } \\
\hline Yes (63) & $7,776(37.4)$ & $6,756(29.8)$ & 121.19 & $<0.0001$ & $49.7 \%$ & Random & $1.09(1.05-1.13)$ & $<0.0001$ \\
\hline No (49) & $1,513(7.3)$ & 3,494 (15.4) & 69.97 & 0.0209 & $31.4 \%$ & Random & $1.15(1.09-1.22)$ & $<0.0001$ \\
\hline NA (104) & $11,508(55.3)$ & $12,430(54.8)$ & 243.89 & $<0.0001$ & $58.2 \%$ & Random & $1.11(1.07-1.14)$ & $<0.0001$ \\
\hline \multicolumn{9}{|l|}{ Gender } \\
\hline Male (25) & $5,072(24.1)$ & $3,883(16.7)$ & 58.69 & 0.0003 & $55.7 \%$ & Random & $1.08(1.02-1.13)$ & 0.0045 \\
\hline Female (26) & $2,221(10.5)$ & $3,165(13.6)$ & 68.93 & $<0.0001$ & $60.8 \%$ & Random & $1.13(1.06-1.22)$ & 0.0003 \\
\hline NA (139) & $13,774(65.4)$ & $16,269(69.8)$ & 249.95 & $<0.0001$ & $46.0 \%$ & Random & $1.11(1.08-1.13)$ & $<0.0001$ \\
\hline
\end{tabular}

Note. Data Included from 170 studies. Only 1 study was Mexican and not counted in subgroup analysis. GSTM1 = Glutathione S transferase mu 1; LC = Lung Cancer; NSCLC = Non-small cell lung cancer; 46 studies had data for both smoker and non-smoker groups; 20 studies had data for both male and female groups; $\mathrm{Q}=$ Cochran's $\mathrm{Q} ; \mathrm{RR}=$ relative risk; $\mathrm{CI}=$ confidence interval; $\mathrm{NA}=$ not available.

inhibiting GST detoxification pathway and on the phase 1 metabolism of cytochrome P4501A promoting the carcinogenic effect and limiting the detoxification property of GSTM1 [35, 36]. Furthermore, the high prevalence of air pollution in China and in other countries may have more impact on the results for smoking and LC risk [3, 35-40]. Future studies can continue to use the standardized risk ratios to see the differences on the risks across different subtypes.

In the gender subgroup analyses, in Southeast Asian subgroup, male gender had higher risk of LC than female gender. For two Southeast Asian studies, male patients had the history of cigarette smoking, tobacco chewing and drinking alcohol $[35,36]$ with more smokers in the LC group (66\%) than the control group (37\%). Possible additional explanation on difference of risk between gender may lay in dietary intake, that quercetin-rich foods taken in South Asians could reduce the risk of LC through overall upregulation of GSTM1, especially for smokers $[19,39]$. Individual studies noted increased GSTM1 deletion in squamous cell carcinoma (SCC) than other LC subtypes [37, 38], in younger and female LC patients [39], and in smokers [2, 40]. A previous meta-analysis for 
Table 3: Meta-prediction: Death from air pollution (APD) on GSTM1 for control (Ct) and lung cancer (LC) cases, and LC risks

\begin{tabular}{|c|c|c|c|c|c|c|c|c|c|c|c|}
\hline \multicolumn{6}{|c|}{ Partition tree } & \multicolumn{6}{|c|}{ Tukey's test } \\
\hline Variable & $\mathrm{AlCc}$ & APD & Count & Mean & SD & $\begin{array}{c}\text { Levels } \\
\text { compared }\end{array}$ & Difference & $\begin{array}{c}\text { SE } \\
\text { Difference }\end{array}$ & $\begin{array}{c}\text { Lower } \\
\text { CI }\end{array}$ & $\begin{array}{c}\text { Upper } \\
\text { CI }\end{array}$ & $p$ \\
\hline Ct Deletion & 1255.60 & $\begin{array}{l}2 \& 3 \\
4\end{array}$ & $\begin{array}{l}89 \\
81\end{array}$ & $\begin{array}{l}45.49 \\
48.65\end{array}$ & $\begin{array}{l}10.52 \\
8.47\end{array}$ & $\begin{array}{l}4 / 3 \\
4 / 2 \\
2 / 3\end{array}$ & $\begin{array}{l}3.2217 \\
3.0779 \\
0.1438\end{array}$ & $\begin{array}{l}1.6917 \\
1.9478 \\
2.0896\end{array}$ & $\begin{array}{l}-0.7790 \\
-1.5285 \\
-4.7978\end{array}$ & $\begin{array}{l}7.2224 \\
7.6842 \\
5.0854\end{array}$ & $\begin{array}{l}0.1406 \\
0.2571 \\
0.9974\end{array}$ \\
\hline LC Deletion & 1241.31 & $\begin{array}{l}2 \& 3 \\
4\end{array}$ & $\begin{array}{l}89 \\
81\end{array}$ & $\begin{array}{l}49.14 \\
55.97\end{array}$ & $\begin{array}{l}9.99 \\
8.25\end{array}$ & $\begin{array}{l}4 / 3 \\
4 / 2 \\
2 / 3\end{array}$ & $\begin{array}{l}7.4552 \\
5.8628 \\
1.5923\end{array}$ & $\begin{array}{l}1.6190 \\
1.8642 \\
1.9998\end{array}$ & $\begin{array}{l}3.6264 \\
1.4544 \\
-3.1370\end{array}$ & $\begin{array}{l}11.2839 \\
10.2713 \\
6.3216\end{array}$ & $\begin{array}{l}<0.0001 \\
0.0056 \\
0.7059\end{array}$ \\
\hline RR Deletion & -24.28 & $\begin{array}{l}2 \& 3 \\
4\end{array}$ & $\begin{array}{l}89 \\
81\end{array}$ & $\begin{array}{l}1.1007 \\
1.1826\end{array}$ & $\begin{array}{l}0.1777 \\
0.2632\end{array}$ & $\begin{array}{l}4 / 3 \\
4 / 2 \\
2 / 3\end{array}$ & $\begin{array}{l}0.0933 \\
0.0642 \\
0.0291\end{array}$ & $\begin{array}{l}0.0391 \\
0.0451 \\
0.0484\end{array}$ & $\begin{array}{l}-0.0007 \\
-0.0424 \\
-0.0853\end{array}$ & $\begin{array}{l}0.1860 \\
0.1709 \\
0.1435\end{array}$ & $\begin{array}{l}0.0479 \\
0.3310 \\
0.8197\end{array}$ \\
\hline \multicolumn{12}{|c|}{ LC Subgroups - NSCLC } \\
\hline Ct Deletion & 284.38 & $\begin{array}{l}2 \& 3 \\
4\end{array}$ & $\begin{array}{l}15 \\
23\end{array}$ & $\begin{array}{l}44.85 \\
48.66\end{array}$ & $\begin{array}{l}12.66 \\
6.99\end{array}$ & $\begin{array}{l}4 / 3 \\
4 / 2 \\
2 / 3\end{array}$ & $\begin{array}{l}5.8817 \\
2.4280 \\
3.4537\end{array}$ & $\begin{array}{l}4.4341 \\
3.8031 \\
5.0979\end{array}$ & $\begin{array}{l}-4.9697 \\
-6.8792 \\
-9.0223\end{array}$ & $\begin{array}{l}16.7332 \\
11.7352 \\
15.9298\end{array}$ & $\begin{array}{l}0.3903 \\
0.8000 \\
0.7780\end{array}$ \\
\hline $\begin{array}{l}\text { LC } \\
\text { Deletion }\end{array}$ & 276.45 & $\begin{array}{l}2 \& 3 \\
4\end{array}$ & $\begin{array}{l}15 \\
23\end{array}$ & $\begin{array}{l}49.21 \\
54.62\end{array}$ & $\begin{array}{l}10.80 \\
6.94\end{array}$ & $\begin{array}{l}4 / 3 \\
4 / 2 \\
2 / 3\end{array}$ & $\begin{array}{l}8.3600 \\
3.4568 \\
4.9032\end{array}$ & $\begin{array}{l}3.9557 \\
3.3928 \\
4.5479\end{array}$ & $\begin{array}{l}-1.3207 \\
-4.8462 \\
-6.2269\end{array}$ & $\begin{array}{l}18.0407 \\
11.7599 \\
16.0332\end{array}$ & $\begin{array}{l}0.1017 \\
0.5701 \\
0.5337\end{array}$ \\
\hline $\begin{array}{l}\text { RR } \\
\text { Deletion }\end{array}$ & -5.90 & $\begin{array}{l}2 \\
3 \& 4\end{array}$ & $\begin{array}{l}9 \\
29\end{array}$ & $\begin{array}{l}1.15 \\
1.14\end{array}$ & $\begin{array}{l}0.29 \\
0.18\end{array}$ & $\begin{array}{l}2 / 3 \\
2 / 4 \\
4 / 3\end{array}$ & $\begin{array}{l}0.0341 \\
0.0150 \\
0.0191\end{array}$ & $\begin{array}{l}0.1125 \\
0.0839 \\
0.0977\end{array}$ & $\begin{array}{l}-0.2413 \\
-0.1904 \\
-0.2204\end{array}$ & $\begin{array}{l}0.3095 \\
0.2204 \\
0.2586\end{array}$ & $\begin{array}{l}0.9507 \\
0.9826 \\
0.9792\end{array}$ \\
\hline $\begin{array}{l}\text { Mixed LC } \\
\mathrm{Ct} \\
\text { Deletion }\end{array}$ & 977.85 & $\begin{array}{l}2 \& 3 \\
4\end{array}$ & $\begin{array}{l}74 \\
58\end{array}$ & $\begin{array}{l}45.61 \\
48.65\end{array}$ & $\begin{array}{l}10.13 \\
9.05\end{array}$ & $\begin{array}{l}4 / 3 \\
4 / 2 \\
3 / 2\end{array}$ & $\begin{array}{l}2.8863 \\
3.3022 \\
0.4160\end{array}$ & $\begin{array}{l}1.8943 \\
2.2913 \\
2.3640\end{array}$ & $\begin{array}{l}-1.6054 \\
-2.1306 \\
-5.1893\end{array}$ & $\begin{array}{l}7.3779 \\
8.7351 \\
6.0213\end{array}$ & $\begin{array}{l}0.2833 \\
0.3229 \\
0.9831\end{array}$ \\
\hline $\begin{array}{l}\text { LC } \\
\text { Deletion }\end{array}$ & 970.32 & $\begin{array}{l}2 \& 3 \\
4\end{array}$ & $\begin{array}{l}74 \\
58\end{array}$ & $\begin{array}{l}49.12 \\
56.50\end{array}$ & $\begin{array}{l}9.90 \\
8.71\end{array}$ & $\begin{array}{l}4 / 3 \\
4 / 2 \\
2 / 3\end{array}$ & $\begin{array}{l}7.7055 \\
6.7632 \\
0.9423\end{array}$ & $\begin{array}{l}1.8401 \\
2.2257 \\
2.2963\end{array}$ & $\begin{array}{l}3.3424 \\
1.4859 \\
-4.5025\end{array}$ & $\begin{array}{c}12.0685 \\
12.0405 \\
6.3871\end{array}$ & $\begin{array}{l}0.0002 \\
0.0080 \\
0.9114\end{array}$ \\
\hline $\begin{array}{l}\text { RR } \\
\text { Deletion }\end{array}$ & -14.01 & $\begin{array}{l}2 \& 3 \\
4\end{array}$ & $\begin{array}{l}74 \\
58\end{array}$ & $\begin{array}{l}1.09 \\
1.20\end{array}$ & $\begin{array}{l}0.16 \\
0.29\end{array}$ & $\begin{array}{l}4 / 3 \\
4 / 2 \\
2 / 3\end{array}$ & $\begin{array}{l}0.1142 \\
0.0915 \\
0.0227\end{array}$ & $\begin{array}{l}0.0442 \\
0.0535 \\
0.0552\end{array}$ & $\begin{array}{l}0.0094 \\
-0.0353 \\
-0.1081\end{array}$ & $\begin{array}{l}0.2191 \\
0.2183 \\
0.1536\end{array}$ & $\begin{array}{l}0.0291 \\
0.2050 \\
0.9107\end{array}$ \\
\hline $\begin{array}{l}\text { Smoker } \\
\mathrm{Ct} \\
\text { Deletion }\end{array}$ & 470.38 & $\begin{array}{l}2 \\
3 \& 4\end{array}$ & $\begin{array}{l}14 \\
49\end{array}$ & $\begin{array}{l}44.03 \\
46.76\end{array}$ & $\begin{array}{l}9.56 \\
9.83\end{array}$ & $\begin{array}{l}4 / 3 \\
4 / 2 \\
3 / 2\end{array}$ & $\begin{array}{l}0.1909 \\
2.8077 \\
2.6168\end{array}$ & $\begin{array}{l}2.8888 \\
3.1891 \\
3.4704\end{array}$ & $\begin{array}{l}-6.7516 \\
-4.8564 \\
-5.7234\end{array}$ & $\begin{array}{c}7.1334 \\
10.4717 \\
10.9569\end{array}$ & $\begin{array}{l}0.9976 \\
0.6547 \\
0.7324\end{array}$ \\
\hline $\begin{array}{l}\mathrm{LC} \\
\text { Deletion }\end{array}$ & 462.77 & $\begin{array}{l}2 \& 3 \\
4\end{array}$ & $\begin{array}{l}33 \\
30\end{array}$ & $\begin{array}{l}45.65 \\
56.35\end{array}$ & $\begin{array}{l}10.91 \\
6.84\end{array}$ & $\begin{array}{l}4 / 3 \\
4 / 2 \\
3 / 2\end{array}$ & $\begin{array}{l}7.8321 \\
14.5944 \\
6.7624\end{array}$ & $\begin{array}{l}2.6206 \\
2.8930 \\
3.1482\end{array}$ & $\begin{array}{l}1.5341 \\
7.6419 \\
-0.8035\end{array}$ & $\begin{array}{l}14.1300 \\
21.5470 \\
14.3282\end{array}$ & $\begin{array}{l}0.0112 \\
<0.0001 \\
0.0888\end{array}$ \\
\hline $\begin{array}{l}\text { RR } \\
\text { Deletion }\end{array}$ & 3.27 & $\begin{array}{l}2 \& 3 \\
4\end{array}$ & $\begin{array}{l}33 \\
30\end{array}$ & $\begin{array}{l}1.01 \\
1.25\end{array}$ & $\begin{array}{l}0.14 \\
0.32\end{array}$ & $\begin{array}{l}4 / 3 \\
4 / 2 \\
3 / 2\end{array}$ & $\begin{array}{l}0.1938 \\
0.2849 \\
0.0910\end{array}$ & $\begin{array}{l}0.0702 \\
0.0775 \\
0.0844\end{array}$ & $\begin{array}{l}0.0250 \\
0.0985 \\
-0.1117\end{array}$ & $\begin{array}{l}0.3626 \\
0.4712 \\
0.2938\end{array}$ & $\begin{array}{l}0.0206 \\
0.0015 \\
0.5308\end{array}$ \\
\hline $\begin{array}{l}\text { Non-smoker } \\
\mathrm{Ct} \\
\text { Deletion }\end{array}$ & 388.52 & $\begin{array}{l}2 \& 3 \\
4\end{array}$ & $\begin{array}{l}22 \\
27\end{array}$ & $\begin{array}{l}42.07 \\
48.32\end{array}$ & $\begin{array}{l}13.86 \\
10.63\end{array}$ & $\begin{array}{l}4 / 3 \\
4 / 2 \\
2 / 3\end{array}$ & $\begin{array}{l}6.5741 \\
5.7677 \\
0.8064\end{array}$ & $\begin{array}{l}4.1549 \\
4.7373 \\
5.3371\end{array}$ & $\begin{array}{l}-3.4884 \\
-5.7054 \\
-12.1191\end{array}$ & $\begin{array}{l}16.6366 \\
17.2407 \\
13.7320\end{array}$ & $\begin{array}{l}0.2635 \\
0.4491 \\
0.9875\end{array}$ \\
\hline $\begin{array}{l}\text { LC } \\
\text { Deletion }\end{array}$ & 401.24 & $\begin{array}{l}2 \\
3 \& 4\end{array}$ & $\begin{array}{l}9 \\
40\end{array}$ & $\begin{array}{l}44.29 \\
56.05\end{array}$ & $\begin{array}{l}10.27 \\
14.50\end{array}$ & $\begin{array}{l}4 / 3 \\
4 / 2 \\
3 / 2\end{array}$ & $\begin{array}{c}4.3969 \\
13.1857 \\
8.7889\end{array}$ & $\begin{array}{l}4.6874 \\
5.3445 \\
6.0211\end{array}$ & $\begin{array}{l}-6.9552 \\
0.2423 \\
-5.7932\end{array}$ & $\begin{array}{l}15.7490 \\
26.1291 \\
23.3709\end{array}$ & $\begin{array}{l}0.6193 \\
0.0450 \\
0.3196\end{array}$ \\
\hline $\begin{array}{l}\text { RR } \\
\text { Deletion }\end{array}$ & 99.91 & $\begin{array}{l}2 \\
3 \& 4\end{array}$ & $\begin{array}{l}9 \\
40\end{array}$ & $\begin{array}{l}1.08 \\
1.31\end{array}$ & $\begin{array}{l}0.24 \\
0.70\end{array}$ & $\begin{array}{l}4 / 2 \\
3 / 4 \\
3 / 2\end{array}$ & $\begin{array}{l}0.1391 \\
0.2993 \\
0.4383\end{array}$ & $\begin{array}{l}0.2441 \\
0.2141 \\
0.2750\end{array}$ & $\begin{array}{l}-0.4522 \\
-0.2193 \\
-0.2278\end{array}$ & $\begin{array}{l}0.7303 \\
0.8178 \\
1.1044\end{array}$ & $\begin{array}{l}0.8369 \\
0.3505 \\
0.2586\end{array}$ \\
\hline
\end{tabular}

Note. AICc $=$ Alkaike's information criterion; APD = Death rates from air pollution levels per million $(2: \leq 100,3: 101-250,4: \geq 251)$, RR $=$ risk ratio; GSTM1 = Glutathione $S$ transferase mu 1; CI = confidence interval.

Chinese populations presented higher risks of LC with GSTM1 deletion for SCC and adenocarcinoma (AC) than the small cell (SC) LC types [2]. A second meta-analysis in Chinese population also reported association of SCC and SC LC than AC subtypes being associated with smoking history [28]. No previous meta-analysis studies 
reported interaction structure or nested structure of LC subtypes with smoking and gender status, rather all studies reported grouping strata of these factors with GSTMI without interaction structure. For LC subtypes, we found similar risks for NSCLC and mixed LC subgroups, with the strata of smoking status according to the data presented
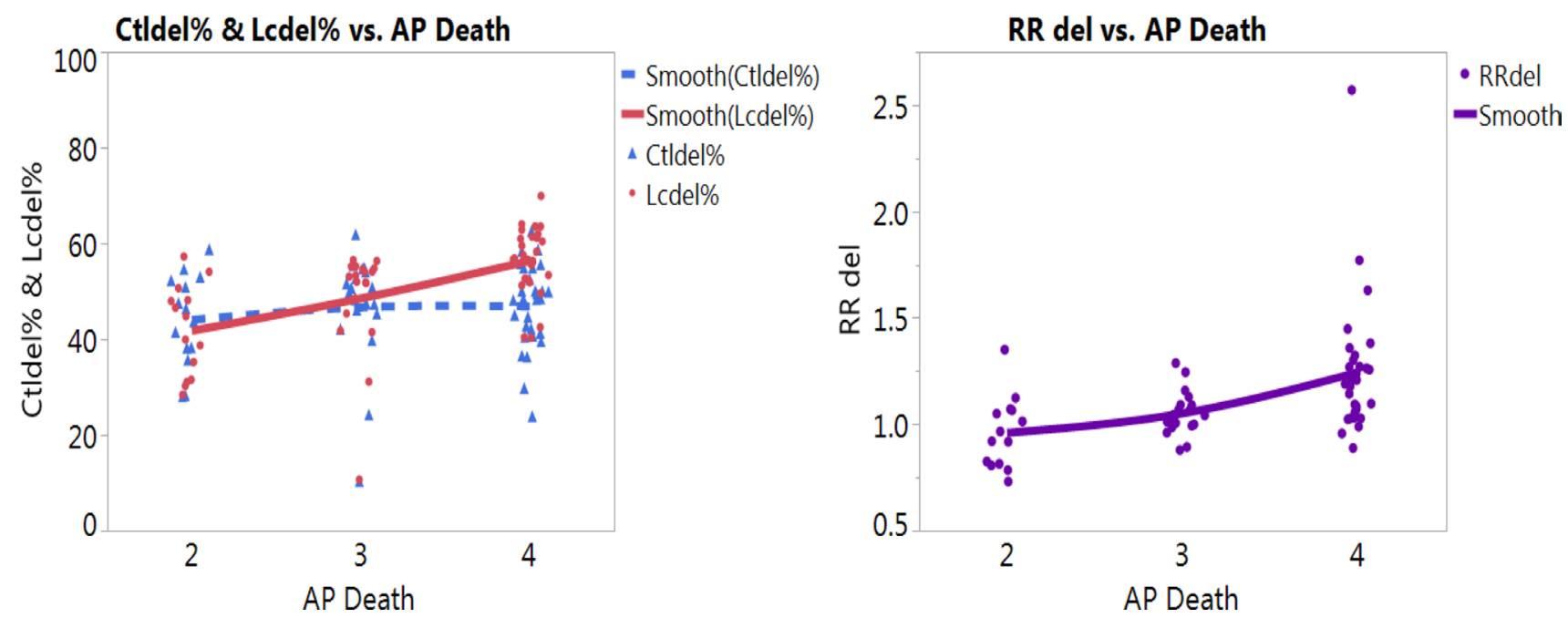

Figure 3: Nonlinear fit on percentages of GSTM1 deletion per control (blue color) and lung cancer (LC, red color) groups (left graph) and LC risk (right graph) with death from air pollution in smokers (AP death: Death from air pollution, Levels per million: $2:<100,3: 101-250,4:>251)$.
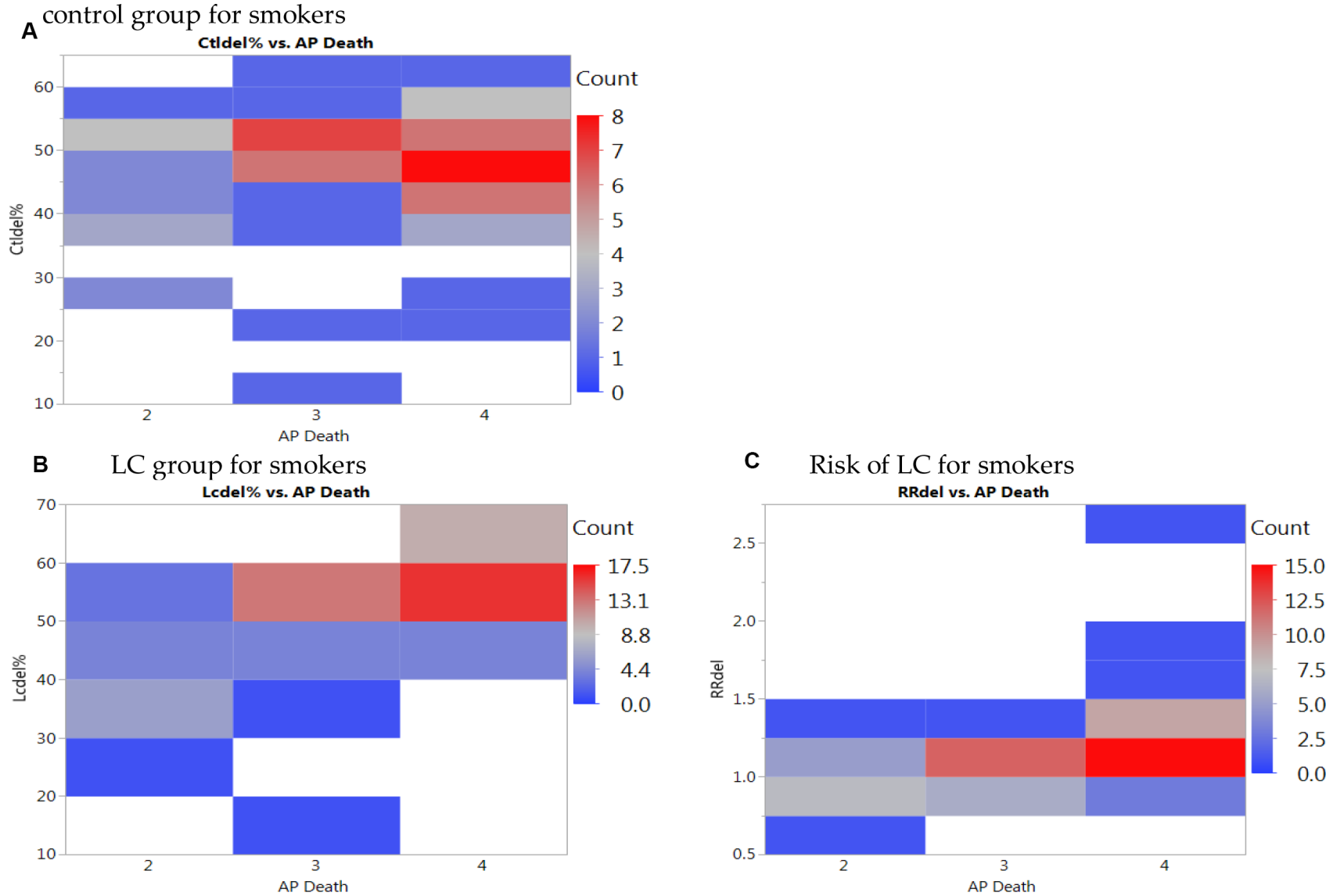

Figure 4: Heat maps of GSTM1 deletion for smokers per (A) control group, (B) lung cancer (LC) group, and (C) LC risk for smokers with death from air pollution (Levels per million: 2: <100, 3:101-250, 4: $>251$ ). 
in the original studies. Future studies are needed to report GSTM1 deletion with interactions of LC subtypes nested with the smoking and gender strata.

Our findings illustrated the complexity of geneenvironment interactions with smoking status across regions and ethnic groups. As the studies on ITC and indole from crucifer-vegetable consumptions showed critical role of micronutrients in detoxification pathway and LC prevention $[14,17-19]$, studies are needed to further identify ways to decrease LC risks in population studies. Specifically, future studies are needed to examine how diet, environmental factors including air pollution and smoking status interact with GSTM1 deletion and polymorphisms across different regions and ethnic groups to prevent LC. Dietary management can be further examined in future intervention studies associating gene-environment interactions for LC prevention. Additionally, future research can be designed to examine other factors for gene environment interactions, such as ITC vegetable consumptions, smoking status, and other risk factors in association with gene-environment interactions for $\mathrm{LC}$ prevention.

Using meta-predictive techniques, we further presented the potential impact of air pollution on increased GSTM1 deletion rates and LC risks. Air pollution played a significant role with increased GSTM1 deletion and LC susceptibility for smokers. In countries with high levels of air pollution (Level 4), for smokers, GSTM1 deletion was a risk to LC susceptibility for most countries except Turkey. Among countries with lower levels of air pollution (Level 2), for smokers, GSTM1 deletion was a risk in Finland and India. From the risk analyses, we found that smoking and increased air pollution had additive effects to the LC risk susceptibilities in addition to the effects of GSTM1 gene polymorphisms on LC risks, for gene-environment interactions (Figure 2).

This meta-analysis should be interpreted within the context of its potential limitations. Limitations of the study include that this study is a population-based study. While we added the effects of air pollution and smoking as possible important contributors of GSTM1 deletion and LC risks, this study is not a study to examine the mechanisms to delineate the interaction effects of air pollution and smoking on GTSM1 deletion and LC. From our metaprediction analysis, we found that air pollution is the most influential factor but not gender and other factors for their effects on GSTM1 polymorphism and LC risks interacting with gene polymorphisms [41-45]. As none of the original individual studies reported the GSTM1 deletion within the interaction or nested contexts of LC subtypes with smoking, we were unable to delineate additional interaction effects for other factors such as gender status with the current meta-analysis data layout. Future studies are needed to accumulate common data elements for the important factors in addition to the gene polymorphisms with a data repository that enables the examination of gene-environment interactions of GSTM1 with various LC subtypes with new emerging interaction analytics [46, 47].

\section{MATERIALS AND METHODS}

\section{Characteristics of original studies}

A literature search was conducted using PubMed database for human studies on LC and GSTM1. The database was periodically searched for latest articles over the course of investigation till 2017, until no additional eligible studies were identified. Additionally, previous metaanalysis and review papers were used to cross reference and trace back to all original studies (See Supplementary References 1-33 following Supplementary Table 1). Of the 163 papers included, additional factors such as gender, smoking status, and types of LC were entered into the database for analysis. Seven papers have data for two racial-ethnic groups for both LC cases and controls [21-26, 48], yielding additional 7 study groups. These studies were conducted around the globe and its continents (e.g., Australia, Europe, North and South America, and Asia). Furthermore, the racial and ethnic composition of each study were checked. The most investigated racialethnic populations for GSTM1 in association with LC was Asian (85 studies), Caucasian (68 studies), African (8 studies), and mixed-race groups (8 studies).

\section{Inclusion and exclusion criteria}

The inclusion criteria were studies that 1) examined the association of GSTM1 and LC risk, reporting the genotype allele counts for both LC cases and controls, 2) were written in English or 3) had abstract written in English with tables of genotype counts that were clearly presented. We excluded studies that 1) were written in non-English languages without genotype counts, 2) did not provide GSTM1 genotype allele counts for LC cases and controls, and 3) were of duplicate studies. Figure 1 presents the study selection process. Of 451 identified potential relevant articles, 240 were excluded as they did not provide GSTM1 genotype counts for LC cases and controls, and 34 were previous meta-analyses. Of the remaining 177 studies, 14 were of duplicate studies (See Supplementary References 34-47 following Supplementary Table 1). At the end, 163 papers, 7 papers with additional subgroups, with appropriate genotype counts were included in the pooled analysis (Figure 1, See Supplementary References 48-210 following Supplementary Table 1).

\section{Quality measures}

Data extractions and entry were checked for accuracy, and systematically organized to identify possible patterns. Preliminary analysis was run to ensure that the ranges of entries and pooled results were accurate for all studies. Each study was evaluated for quality using a set of appropriate indicators adapted from multiple sources on the assessment of studies. Integrated sources for these criteria included the 
U.S. QUOROM consensus process on the quality of metaanalysis [49], quality reporting for observational studies $[50,51]$, and in recent studies using the similar analytics $[45,52]$. Details on quality indicators that were used to assess the studies were presented in Supplementary Table 1 . The total range of quality score was $0-30$ based on three domains: 1) external validity with 10 items on demographic factors (score range of $0-11$ ); 2) internal validity with 12 items on methods and procedures (score range of $0-12$ ); and, 3) report quality with 7 items on study results (score range of 0-7) [52]. The total quality score of included studies ranged from 8 to 28 (out of 30 maximum score). Studies scored above $50 \%$ for the possible total score were judged to have trustworthy findings [49]. We included all studies as we did not observe differences with pooled analyses when studies with low quality scores were analyzed in separate groups for sensitivity analyses.

\section{Data synthesis and analysis}

We entered the air-quality data for various countries. Specifically, we verified from various sources for the most current and complete air-pollution data including the death rates from air pollution (death rates per million, Level 1: 50, Level 2: 51-100, Level 3: 101-250, Level 4: 251-400, Level 5: $\geq 401[53,54]$. We further verified these levels with current scales on air pollution data [55-58], and the most complete and current data on air pollution data was used for the analyses. There were no studies with Levels 1 or 5 pollution, therefore only Levels 2-4 pollution were included for final analysis.

Prior to analyses, we entered all data into Excel spreadsheets (Microsoft Corp, Redmond, WA). HardyWeinberg Equilibrium (HWE) analyses were checked, which was developed to assess the distribution equilibrium for the evolutionary mechanisms on the population genetics $[59,60]$. Departure from the HWE with a $p$ value $P<0.05$ may be associated with factors such as population migration or stratification, and disease association. The associations of GSTM1 deletion with LC risk was estimated by calculating pooled risk ratio (RR)s and 95\% CI between cases and controls, using StatsDirect version 3.0 software (Cheshire, UK). Pooled RR has been used in most recent consensus reports for standardized risk ratios for more conservative reports and for standardization across all factors included in the geneenvironment interaction analysis [20].

We utilized JMP pro 13 program (SAS Institute, Cary, NC) for meta-prediction analysis to examine the association of air pollution associated death (APD) to GSTM1 deletion polymorphisms and LC risk. We used partition tree to examine the association between independent and dependent variables. The "goodness of the partition" can be judged using Akaike's information criterion (AIC) or AIC with correction (AICc), in which a smaller AIC or AICc suggests a better model $[52,61]$.
AIC is a fitness index for trading off the complexity of a model against how well the model fits the data. Increasing the number of free parameters to be estimated improves the model fitness, however, the model might be unnecessarily complex. To reach a balance between fitness and parsimony, the "best" model is the one with the lowest AIC value. In this sense, AIC is better than $\mathrm{R}^{2}$ and adjusted $\mathrm{R}^{2}$ used in meta-regression [20], which always go up as additional variables enter in the model, favoring complexity. However, AIC does not necessarily change by adding variables. Rather, it varies based upon the composition of the predictors and thus it is a better indicator of the model quality.

Additionally, we used nonlinear fit, heat maps, and Tukey's posthoc test to further validate meta-prediction findings. In particular, we used Tukey's tests to compare AICc results with the partition trees [62]. All $p$ values were two-tailed with a significant level at $P<0.05$. GIS maps was prepared to better visualize the heterogeneity of GSTM1 deletion polymorphism with LC risks on the world map. We applied meta-predictive analytical techniques using recursive partition tree, nonlinear fit and heat maps for data visualization to reveal nonlinear patterns in this study, in addition to the conventional pooled-analysis technique, to visualize the heterogeneity. While meta-regression is used commonly for advanced meta-analysis for meta-prediction [20], it is important to point out that regression analysis, as a linear model, is unable to detect nonlinear patterns. Further, it is well known that regression based on $\mathrm{R}^{2}$ tends to yield a complex and overfitted model because $\mathrm{R}^{2}$ always goes up with additional predictors. On the other hand, AIC or AICc does not necessarily change with the addition of variables. Rather, it varies based upon the composition of the predictors; thus, it is more likely to yield an optimal model [63-65].

\section{Author contributions}

Conceived the concepts: Pojui Yu and Shyang-Yun Pamela Koong Shiao; Wrote the first draft of the manuscript: Pojui Yu, Joyce D. Kusuma, Maria Aurora R. Suarez and Shyang-Yun Pamela Koong Shiao; Data analysis: Pojui Yu, Joyce D. Kusuma, and Shyang-Yun Pamela Koong Shiao; Agreed with manuscript results and conclusions: all authors reviewed and approved of the final manuscript. This metaanalysis was registered with PROSPERO, International prospective register of systematic reviews, number: 96460 at https://www.crd.york.ac.uk/PROSPERO/\#myprospero; http://prisma-statement.org/Protocols/Registration. There was no prior study registered on this subject in this registry.

\section{ACKNOWLEDGMENTS}

The authors acknowledge the help from Amanda Lie with finding literature. 


\section{CONFLICTS OF INTEREST}

The authors declare no conflicts of interest.

\section{FUNDING}

Funding support includes the Doctoral Research Council Grants, Azusa Pacific University; Research Start-up fund from Augusta University awarded to the corresponding author; and $\mathrm{Fu}$ Jen University Hospital, Taiwan, awarded to the first author.

\section{REFERENCES}

1. ACS. Cancer Facts \& Figures 2018. Available online: https:// www.cancer.org/content/dam/cancer-org/research/cancer-factsand-statistics/annual-cancer-facts-and-figures/2018/cancerfacts-and-figures-2018.pdf (accessed on 18 March 2018).

2. Yang H, Yang S, Liu J, Shao F, Wang H, Wang Y. The association of GSTM1 deletion polymorphism with lung cancer risk in Chinese population: evidence from an updated meta-analysis. Sci Rep. 2015; 5:9392. https://doi. org/10.1038/srep09392.

3. Zhang H, Wu X, Xiao Y, Chen M, Li Z, Wei X, Tang K. Genetic polymorphisms of glutathione S-transferase M1 and $\mathrm{T} 1$, and evaluation of oxidative stress in patients with non-small cell lung cancer. Eur J Med Res. 2014; 19:67-72. https://doi.org/10.1186/s40001-014-0067-3.

4. Carlsten C, Sagoo GS, Frodsham AJ, Burke W, Higgins JP. Glutathione S-transferase M1 (GSTM1) polymorphisms and lung cancer: a literature-based systematic HuGE review and meta-analysis. Am J Epidemiol. 2008; 167:759-74. https://doi.org/10.1093/aje/kwm383.

5. López-Cima MF, Alvarez-Avellón SM, Pascual T, Fernández-Somoano A, Tardón A. Genetic polymorphisms in CYP1A1, GSTM1, GSTP1 and GSTT1 metabolic genes and risk of lung cancer in Asturias. BMC Cancer. 2012; 12:433-39. https://doi.org/10.1186/1471-2407-12-433.

6. Mota P, Silva HC, Soares MJ, Pego A, Loureiro M, Cordeiro CR, Regateiro FJ. Genetic polymorphisms of phase I and phase II metabolic enzymes as modulators of lung cancer susceptibility. J Cancer Res Clin Oncol. 2015; 141:851-60. https://doi.org/10.1007/s00432-014-1868-z.

7. NCBI. GSTM1 glutathione S-transferase mu [Homo sapiens (human)]. Available online: http://www.ncbi.nlm.nih.gov/ gene/2944 (accessed on 18 January 2018).

8. Sharma N, Singh A, Singh N, Behera D, Sharma S. Genetic polymorphisms in GSTM1, GSTT1 and GSTP1 genes and risk of lung cancer in a North Indian population. Cancer Epidemiol. 2015; 39:947-55. https://doi.org/10.1016/j. canep.2015.10.014.

9. Ketterer B, Coles B, Meyer DJ. The role of glutathione in detoxication. Environ Health Perspect. 1983; 49:59-69. https://doi.org/10.1289/ehp.834959.
10. Moyer AM, Sun Z, Batzler AJ, Li L, Schaid DJ, Yang P, Weinshilboum RM. Glutathione pathway genetic polymorphisms and lung cancer survival after platinum-based chemotherapy. Cancer Epidemiol Biomarkers Prev. 2010; 19:811-21. https://doi.org/10.1158/1055-9965.EPI-09-0871.

11. Kalinina EV, Chernov NN, Novichkova MD. Role of glutathione, glutathione transferase, and glutaredoxin in regulation of redox-dependent processes. Biochemistry (Mosc). 2014; 79:1562-83. https://doi.org/10.1134/ S0006297914130082.

12. Seidegård J, Pero RW, Miller DG, Beattie EJ. A glutathione transferase in human leukocytes as a marker for the susceptibility to lung cancer. Carcinogenesis. 1986; 7:75153. https://doi.org/10.1093/carcin/7.5.751.

13. Cadet J, Douki T, Ravanat JL. Oxidatively generated base damage to cellular DNA. Free Radic Biol Med. 2010; 49:921. https://doi.org/10.1016/j.freeradbiomed.2010.03.025.

14. Fowke JH, Gao YT, Chow WH, Cai Q, Shu XO, Li HL, Ji BT, Rothman N, Yang G, Chung FL, Zheng W. Urinary isothiocyanate levels and lung cancer risk among non-smoking women: a prospective investigation. Lung Cancer. 2011; 73:18-24. https://doi.org/10.1016/j.lungcan.2010.10.024.

15. CDC. Lung cancer. Available online: http://www.cdc.gov/ cancer/lung/basic_info/index.htm (accessed on 18 January 2018).

16. Hair JM, Terzoudi GI, Hatzi VI, Lehockey KA, Srivastava D, Wang W, Pantelias GE, Georgakilas AG. BRCA1 role in the mitigation of radiotoxicity and chromosomal instability through repair of clustered DNA lesions. Chem Biol Interact. 2010; 188:350-58. https://doi. org/10.1016/j.cbi.2010.03.046.

17. Zhao B, Seow A, Lee EJ, Poh WT, Teh M, Eng P, Wang YT, Tan WC, Yu MC, Lee HP. Dietary isothiocyanates, glutathione S-transferase -M1, -T1 polymorphisms and lung cancer risk among Chinese women in Singapore. Cancer Epidemiol Biomarkers Prev. 2001; 10:1063-67.

18. Yuan JM, Murphy SE, Stepanov I, Wang R, Carmella SG, Nelson HH, Hatsukami D, Hecht SS. 2-phenethyl isothiocyanate, Glutathione S-transferase M1 and T1 polymorphisms, and detoxification of volatile organic carcinogens and toxicants in tobacco smoke. Cancer Prev Res (Phila). 2016; 9:598-606. https://doi. org/10.1158/1940-6207.CAPR-16-0032.

19. Lam TK, Gallicchio L, Lindsley K, Shiels M, Hammond E, Tao XG, Chen L, Robinson KA, Caulfield LE, Herman JG, Guallar E, Alberg AJ. Cruciferous vegetable consumption and lung cancer risk: a systematic review. Cancer Epidemiol Biomarkers Prev. 2009; 18:184-95. https://doi. org/10.1158/1055-9965.EPI-08-0710.

20. Deeks JJ, Higgins JP, Altman DG. Analysing data and undertaking meta-analyses. In: Higgins JP, Green S, editors. Cochrane Handbook for Systematic Reviews of Interventions: Cochrane Book Series. Chichester, UK: John Wiley \& Sons, Ltd.; 2008. https://doi.org/10.1002/9780470712184.ch9. 
21. Cote ML, Kardia SL, Wenzlaff AS, Land SJ, Schwartz AG. Combinations of glutathione S-transferase genotypes and risk of early-onset lung cancer in Caucasians and African Americans: a population-based study. Carcinogenesis. 2005; 26:811-19. https://doi.org/10.1093/carcin/bgi023.

22. Cote ML, Yoo W, Wenzlaff AS, Prysak GM, Santer SK, Claeys GB, Van Dyke AL, Land SJ, Schwartz AG. Tobacco and estrogen metabolic polymorphisms and risk of nonsmall cell lung cancer in women. Carcinogenesis. 2009; 30:626-35. https://doi.org/10.1093/carcin/bgp033.

23. Wenzlaff AS, Cote ML, Bock CH, Land SJ, Schwartz AG. GSTM1, GSTT1 and GSTP1 polymorphisms, environmental tobacco smoke exposure and risk of lung cancer among never smokers: a population-based study. Carcinogenesis. 2005; 26:395-401. https://doi.org/10.1093/ carcin/bgh326.

24. Kelsey KT, Spitz MR, Zuo ZF, Wiencke JK. Polymorphisms in the glutathione S-transferase class mu and theta genes interact and increase susceptibility to lung cancer in minority populations (Texas, United States). Cancer Causes Control. 1997; 8:554-59. https://doi.org/10.1023/A:1018434027502.

25. London SJ, Daly AK, Cooper J, Navidi WC, Carpenter CL, Idle JR. Polymorphism of glutathione S-transferase M1 and lung cancer risk among African-Americans and Caucasians in Los Angeles County, California. J Natl Cancer Inst. 1995; 87:1246-53. https://doi.org/10.1093/jnci/87.16.1246.

26. London SJ, Daly AK, Leathart JB, Navidi WC, Idle JR. Lung cancer risk in relation to the CYP2C9*1/CYP2C9*2 genetic polymorphism among African-Americans and Caucasians in Los Angeles County, California. Pharmacogenetics. 1996; 6:527-33. https://doi.org/10.1097/00008571-199612000-00006.

27. Chen XP, Xu WH, Xu DF, Xie XH, Yao J, Fu SM. GSTM1 polymorphisms and lung cancer risk in the Chinese population: a meta-analysis based on 47 studies. Asian Pac J Cancer Prev. 2014; 15:7741-46. https://doi.org/10.7314/ APJCP.2014.15.18.7741.

28. Liu K, Lin X, Zhou Q, Ma T, Han L, Mao G, Chen J, Yue X, Wang H, Zhang L, Jin G, Jiang J, Zhao J, Zou B. The associations between two vital GSTs genetic polymorphisms and lung cancer risk in the Chinese population: evidence from 71 studies. PLoS One. 2014; 9:e102372. https://doi. org/10.1371/journal.pone.0102372.

29. How AICR Recommendations Cuts Colorectal Cancer Risk for Both Men and Women. Available online: http:// www.aicr.org/cancer-research-update/2016/11_02/cru-howAICR-recommendations-cuts-colorectal-cancer-risk-formen-and-women.html (accessed 18 January 2018).

30. Viera AJ. Odds ratios and risk ratios: what's the difference and why does it matter? South Med J. 2008; 101:730-34. https://doi.org/10.1097/SMJ.0b013e31817a7ee4.

31. Horgan AM, Yang B, Azad AK, Amir E, John T, Cescon DW, Wheatley-Price P, Hung RJ, Shepherd FA, Liu G. Pharmacogenetic and germline prognostic markers of lung cancer. J Thorac Oncol. 2011; 6:296-304. https://doi. org/10.1097/JTO.0b013e3181ffe909.
32. Langevin SM, Ioannidis JP, Vineis P, Taioli E, and Genetic Susceptibility to Environmental Carcinogens group (GSEC). Assessment of cumulative evidence for the association between glutathione S-transferase polymorphisms and lung cancer: application of the Venice interim guidelines. Pharmacogenet Genomics. 2010; 20:586-97. https://doi. org/10.1097/FPC.0b013e32833c3892.

33. Vineis P, Anttila S, Benhamou S, Spinola M, Hirvonen A, Kiyohara C, Garte SJ, Puntoni R, Rannug A, Strange $\mathrm{RC}$, Taioli E. Evidence of gene gene interactions in lung carcinogenesis in a large pooled analysis. Carcinogenesis. 2007; 28:1902-05. https://doi.org/10.1093/carcin/bgm039.

34. Liu H, Ma HF, Chen YK. Association between GSTM1 polymorphisms and lung cancer: an updated metaanalysis. Genet Mol Res. 2015; 14:1385-92. https://doi. org/10.4238/2015.February.13.17.

35. Shah PP, Singh AP, Singh M, Mathur N, Mishra BN, Pant MC, Parmar D. Association of functionally important polymorphisms in cytochrome $\mathrm{P} 4501 \mathrm{~B} 1$ with lung cancer. Mutat Res. 2008; 643:4-10. https://doi.org/10.1016/j. mrfmmm.2008.05.001.

36. Lam TK, Rotunno M, Lubin JH, Wacholder S, Consonni D, Pesatori AC, Bertazzi PA, Chanock SJ, Burdette L, Goldstein AM, Tucker MA, Caporaso NE, Subar AF, Landi MT. Dietary quercetin, quercetin-gene interaction, metabolic gene expression in lung tissue and lung cancer risk. Carcinogenesis. 2010; 31:634 42. https://doi.org/10.1093/carcin/bgp334.

37. Zheng D, Hua F, Mei C, Wan H, Zhou Q. [Association between GSTM1 genetic polymorphism and lung cancer risk by SYBR green I real-time PCR assay]. [Article in Chinese]. Zhongguo Fei Ai Za Zhi. 2010; 13:506-10. https://doi.org/10.3779/j.issn.1009-3419.2010.05.23.

38. Hou SM, Ryberg D, Fält S, Deverill A, Tefre T, Børresen AL, Haugen A, Lambert B. GSTM1 and NAT2 polymorphisms in operable and non-operable lung cancer patients. Carcinogenesis. 2000; 21:49-54. https://doi. org/10.1093/carcin/21.1.49.

39. Kihara M, Noda K, Kihara M. Distribution of GSTM1 null genotype in relation to gender, age and smoking status in Japanese lung cancer patients. Pharmacogenetics. 1995; 5:S74 79. https://doi.org/10.1097/00008571-199512001-00005.

40. Schoket B, Phillips DH, Kostic S, Vincze I. Smokingassociated bulky DNA adducts in bronchial tissue related to CYP1A1 MspI and GSTM1 genotypes in lung patients. Carcinogenesis. 1998; 19:841-46. https://doi.org/10.1093/ carcin/19.5.841.

41. Wu SM, Chen ZF, Young L, Shiao SP. Meta-Prediction of the Effect of Methylenetetrahydrofolate Reductase Polymorphisms and Air Pollution on Alzheimer's Disease Risk. Int J Environ Res Public Health. 2017; 14. https://doi. org/10.3390/ijerph14010063.

42. Lien SA, Young L, Gau BS, K Shiao SP. Meta-prediction of MTHFR gene polymorphism-mutations, air pollution, and risks of leukemia among world populations. Oncotarget. 2017; 8:4387-98. https://doi.org/10.18632/oncotarget.13876. 
43. Gonzales MC, Yu PJ, Shiao SP. MTHFR Gene PolymorphismMutations and Air Pollution as Risk Factors for Breast Cancer: A Metaprediction Study. Nurs Res. 2017; 66:152-63. https:// doi.org/10.1097/NNR.0000000000000206.

44. Yang YL, Yang HL, Shiao SPK. Meta-Prediction of MTHFR Gene Polymorphisms and Air Pollution on the Risk of Hypertensive Disorders in Pregnancy Worldwide. Int J Environ Res Public Health. 2018; 15. https://doi. org/10.3390/ijerph15020326.

45. Kennedy DA, Stern SJ, Matok I, Moretti ME, Sarkar M, Adams-Webber T, Koren G. Folate intake, MTHFR polymorphisms, and the risk of colorectal cancer: A systematic review and meta-analysis. J Cancer Epidemiol. 2012; 2012:952508. https://doi.org/10.1155/2012/952508.

46. Shiao SP, Grayson J, Yu CH, Wasek B, Bottiglieri T. Gene Environment Interactions and Predictors of Colorectal Cancer in Family-Based, Multi-Ethnic Groups. J Pers Med. 2018; 8. https://doi.org/10.3390/jpm8010010.

47. Gonzales MC, Grayson J, Lie A, Yu CH, Shiao SPK. Geneenvironment interactions and predictors of breast cancer in family-based multi-ethnic groups. Oncotarget. 2018; 9:29019-35. https://doi.org/10.18632/oncotarget.25520.

48. Cabral RE, Caldeira-de-Araujo A, Cabral-Neto JB, Costa Carvalho MG. Analysis of GSTM1 and GSTT1 polymorphisms in circulating plasma DNA of lung cancer patients. Mol Cell Biochem. 2010; 338:263-69. https://doi. org/10.1007/s11010-009-0360-6.

49. Moher D, Liberati A, Tetzlaff J, Altman DG, and PRISMA Group. Preferred reporting items for systematic reviews and meta-analyses: the PRISMA statement. J Clin Epidemiol. 2009; 62:1006-12. https://doi.org/10.1016/j.jclinepi.2009.06.005.

50. Downs SH, Black N. The feasibility of creating a checklist for the assessment of the methodological quality both of randomised and non-randomised studies of health care interventions. J Epidemiol Community Health. 1998; 52:377-84. https://doi.org/10.1136/jech.52.6.377.

51. Stroup DF, Berlin JA, Morton SC, Olkin I, Williamson GD, Rennie D, Moher D, Becker BJ, Sipe TA, Thacker SB. Meta-analysis of observational studies in epidemiology: a proposal for reporting. Meta-analysis Of Observational Studies in Epidemiology (MOOSE) group. JAMA. 2000; 283:2008-12. https://doi.org/10.1001/jama.283.15.2008.

52. Shiao SP, Yu CH. Meta-Prediction of MTHFR Gene Polymorphism Mutations and Associated Risk for Colorectal Cancer. Biol Res Nurs. 2016; 18:357-69. https:// doi.org/10.1177/1099800415628054.
53. US environmental protection agency, air quality index basics. Available online: http://www.airnow.gov/index. cfm?action=aqibasics.aqi (accessed 18 January 2018).

54. Kenworthy J, Laube F. Urban transport patterns in a global sample of cities and their linkages to transport infrastructure, land use, economics and environment. World Transport Policy \& Practice 2002; 8:5-19. ISSN: 1352-7614.

55. WHO. Deaths attributable to urban air pollution. Available online: http://www.who.int/heli/risks/urban/en/ uapmap.1.pdf?ual/41 (accessed 18 January 2018).

56. WHO. Global health risks. Available online: http://www.who. int/healthinfo/global_burden_disease/GlobalHealthRisks_ report_full.pdf (accessed 18 January 2018).

57. WHO. Global health risks. Available online: https://commons. wikimedia.org/wiki/File:Deaths_from_air_pollution.png (accessed 18 January 2018).

58. WHO. The urban environment. Available online: http://www. who.int/heli/risks/urban/urbanenv/en/ (accessed 18 January 2018).

59. Sha Q, Zhang S. A test of Hardy-Weinberg equilibrium in structured populations. Genet Epidemiol. 2011; 35:671-78. https://doi.org/10.1002/gepi.20617.

60. Wittke-Thompson JK, Pluzhnikov A, Cox NJ. Rational inferences about departures from Hardy-Weinberg equilibrium. Am J Hum Genet. 2005; 76:967-86. https:// doi.org/10.1086/430507.

61. Akaike H. Akaike's Information Criterion. In: Lovric M, editor. International Encyclopedia of Statistical Science. Berlin \& Heidelberg, Germany: Springer; 2011. https://doi. org/10.1007/978-3-642-04898-2_110.

62. Jaccard J, Becker MA, Wood G. Pairwise multiple comparison procedures: A review. Psychol Bull. 1984; 96:589-96. https://doi.org/10.1037/0033-2909.96.3.589.

63. Albrecht J. Key Concepts and Techniques in GIS. Sage. 2007. https://doi.org/10.4135/9780857024442.

64. Vanitha A, Niraimathi S. Study on decision tree competent data classification. International Journal of Computer Science and Mobile Computing (IJCSMC) 2013; 2:365-70. ISSN 2320-088X.

65. Faraway JJ. Extending the Linear Model with R: Generalized Linear, Mixed Effects and Nonparametric Regression Models, Second Edition. Chapman \& Hall/ CRC, Taylor and Frances Group: 2016. 